\title{
A Cross-Sectional, Multicentric Clinico- Epidemiological Study of Melasma in India
}

\author{
Devasthanam Sundara Rao KrupaShankar • Vijay Kumar Somani • Malvika Kohli • Jaishree Sharad • \\ Anil Ganjoo • Sanjiv Kandhari • Venkat Ram Mysore • Sanjeev Aurangabadkar • Subrata Malakar • \\ Maya Vedamurthy $\cdot$ Ganesh Kadhe $\cdot$ Salman Motlekar $\cdot$ Pashmina Ahirrao
}

To view enhanced content go to www.dermtherapy-open.com

Received: January 17, 2014 / Published online: March 19, 2014

(C) The Author(s) 2014. This article is published with open access at Springerlink.com

\section{ABSTRACT}

Background: Melasma is one of the most common pigment disorders seen by a dermatologist and often occurs among women with darker complexion (skin type IV-VI).

Aims: The present study aimed to investigate the epidemiology of melasma in the Indian

Electronic supplementary material The online version of this article (doi:10.1007/s13555-014-0046-1) contains supplementary material, which is available to authorized users.

D. S. R. KrupaShankar ( $\square)$

Department of Dermatology, Manipal Hospital,

Bangalore 560017, Karnataka, India

e-mail: dermakrupa@yahoo.com

V. K. Somani

Somani Skin and Cosmetology Centre Clinic,

Himayathnagar, Hyderabad, Andhra Pradesh, India

M. Kohli

306/403/408 Doctor House, 14 Peddar Road,

Mumbai 400026, Maharashtra, India

J. Sharad

Skinfiniti Skin and Laser Clinic, Linking Road,

Bandra (W), Mumbai, Maharashtra, India

A. Ganjoo

Skin and Cosmetology Centre, Gujrawala Town,

Delhi (North) 110009, New Delhi, India

S. Kandhari

Munirka Marg, Vasant Vihar, New Delhi, India population and to focus on the regional variability in the demographics, clinical manifestations and factors that precipitate this condition.

Methods: The present multicentric study conducted across four regions in India enrolled patients ( $>18$ years) diagnosed with melasma on Wood's light examination. Patients were examined to identify the distribution of melasma. Various precipitating

\author{
V. R. Mysore \\ Venkat Charmalaya Clinic, Vijayanagar, Bangalore, \\ Karnataka, India \\ S. Aurangabadkar \\ Skin and Laser Clinic, Brij Tarang, Green Lands, \\ Begumpet, Hyderabad, Andhra Pradesh, India \\ S. Malakar \\ Rita Skin Foundation, GD Block, Salt Lake City, \\ Kolkata 700106, West Bengal, India \\ M. Vedamurthy \\ RSV Skin and Laser Centre, Mahalinghapuram, \\ Chennai, Tamil Nadu, India \\ G. Kadhe $\cdot$ S. Motlekar $\cdot$ P. Ahirrao \\ Wockhardt Ltd., Wockhardt Towers, Bandra Kurla \\ Complex, Mumbai 400051, Maharashtra, India
}


and etiological factors for melasma were documented.

Results: The mean age of the 331 enrolled patients with melasma was $37.2 \pm 9.3$ years. The prevalence of melasma was higher in females with a female to male ratio of approximately $4: 1$. The overall population with family history was $31 \%$, highest in the northern region (38.5\%) and lowest in the eastern region (18.2\%). The two prominent patterns of distribution were centrofacial (42\%) and malar (39\%). Only 35\% of the patients were using sunscreens. Of these, $10 \%$ of the patients used sunscreen with SPF $>50$. The usage of sunscreens was observed to be highest in the north (69\%). About 51\% of women with multiple pregnancies had a history of melasma when compared with single women $(25 \%)$ or with no pregnancy (24\%).

Conclusions: In conclusion, the result of the study showed that there was a regional variability in the demographics, clinical manifestations and factors that precipitate melasma among patients in India. There was a strong correlation between the family history and prevalence of melasma. Sun exposure is a major precipitating factor in melasma, but only $10 \%$ of the patients used sunscreen with SPF $>50$. Other factors such as concomitant medication, chronicity of disease, multiple pregnancies and use of oral contraceptives might precipitate melasma.

Keywords: Dermatology; Epidemiology; Etiology; Melasma; India

\section{INTRODUCTION}

Melasma occurs due to an increased melanogenesis in the melanocytes [1]. Melasma is common among women with darker complexion (skin type IV-VI) [2].
Although the prevalence of melasma has not been investigated in most countries, melasma accounts for about $4-10 \%$ of new cases in dermatology hospitals [3, 4].

Melasma is a common pigmentation disorder among Indians [5]. Malar pattern melasma is predominant in the southern region of India to a greater extent when compared with the northern region [6-8]. Achar et al. demonstrated that an increased exposure to sun, pregnancy, use of oral contraceptives and use of cosmetics at least five times a week contribute to the exacerbation of melasma among the Indian population [7]. The effect of socioeconomic class of the patient, effect of previous treatments, menopause status and other concomitant conditions have not been evaluated in epidemiological studies conducted to evaluate the prevalence of melasma [9-11].

The present study aimed to investigate the epidemiology of melasma in Indian patients using a multicenter collaboration study and to focus on the regional variability in the demographics, clinical manifestations and factors that precipitate this condition.

\section{METHODS}

\section{Study Characteristics}

The present cross-sectional, multicentric study was conducted at nine study centers (Bangalore 2, Hyderabad 2, Delhi 2, Kolkata 1 and Mumbai 2) across four regions (north, south, east and West) in India over a period of 6 months (March 2012-August 2012).

The study protocol and all the amendments were reviewed and approved by an independent institutional ethics committee/institutional review board (IEC/IRB). The study was conducted in accordance with good clinical 
practice (GCP) as required by the International Conference on Harmonization (ICH) guidelines and adopted in Schedule Y (Amended 2005) and also followed the Helsinki Declaration of 1975, as revised in 2000 and 2008. Informed consent was obtained from all patients for being included in the study.

\section{Inclusion and Exclusion Criteria}

All male and female patients ( $>18$ years) diagnosed with melasma on Wood's light examination were included in the present study. Employees of the investigator or those directly involved in the study or other similar trials were excluded from the study. Patients who were unable to provide accurate information regarding the condition were also excluded.

\section{Informed Consent}

All patients were required to provide a written informed consent approved by IRB/IEC for participation in the study.

\section{Study Assessments}

All patients were assessed for demographics including age, gender, marital status, geographical location, and family history (primary relative with melasma). Patients were examined to identify the distribution of melasma which was divided into four regions: centrofacial (cheeks, forehead, upper lip, nose and chin); malar (cheeks and nose); mandibular (ramus of the mandible); and combination patterns (centrofacial and malar, centrofacial and mandibular, malar and mandibular, centrofacial, malar and mandibular).

In addition, the age of onset, disease chronicity ( $<1$ year to $>3$ years), cosmetic usage (including frequency and duration), prior treatments used for melasma, etiological factors including sun exposure, use of sunscreen, pregnancy history, use of hormone replacement therapy (HRT) among postmenopausal women, oral contraceptives use and concomitant conditions were documented. All the patients were examined by a board-certified dermatologist.

\section{Data Collection}

Data of all patients were collected in electronic case report forms (e-CRFs). The completed e-CRFs were submitted and locked for review. All e-CRFs were reviewed by the principal investigator to ensure entry of correct information. The e-CRFs were also verified with the source documents for consistency.

\section{Statistical Analysis}

The mean age of all the patients was calculated using descriptive statistics. One-way analysis of variance (ANOVA) was performed to determine the statistical difference of mean age of patients across all regions. Tukey's post hoc analysis, a single-step, multiple comparison procedure performed in conjunction with ANOVA was used to determine the difference in region with age groups. The mean exposure for each pattern of melasma was obtained and evaluated for statistical significance using one-way ANOVA. A $p<0.05$ was considered as statistically significant.

\section{RESULTS}

This was a time-bound study and not number defined and only those with a diagnosis of melasma were enumerated and studied. A total of 331 patients with a mean age of 
$37.2 \pm 9.3$ years (range 20-62 years) with melasma were included in the study. The male ( $n$-65) to female ( $n$-266) ratio was 1:4; however, the difference in ratio across the regions was not significant $(p=0.5604)$ (Table 1$)$.

\section{Family History, Gender, and Age of Onset}

Overall, a family history of melasma was observed in $31.1 \%$ of the patients (Table 1).In patients with melasma, family history and gender did not show any association $(p>0.05)$. The proportion of the patients with family history of melasma was highest in the north $(38.5 \%)$ and lowest in the east $(18.2 \%)$ region. The mean age of onset of melasma was 34.1 years (east 31.2 years; west 35.2 years; north 37.2 years and south 32.5 years) and most of the patients were diagnosed with melasma between the age of 30 and 39 years. (Table 2). The age of onset was not significantly associated with the family history of melasma $(p=0.5804)$.

\section{Disease Chronicity}

About $43 \%$ of the patients had a disease chronicity of $>3$ years. Overall, $52 \%(54 / 103)$ of the patients with a family history had disease chronicity of $>3$ years, as against $39 \%(88 / 228)$ of the patients, who did not have a history, but had chronicity of $>3$ years $(p=0.0539)$.

\section{Pattern and Type of Melasma}

The predominant patterns observed in the patients was centrofacial melasma (42\%) followed by malar (39\%) melasma (Table 2). A combination of centrofacial, malar and mandibular melasma was observed in $2 \%$ of the patients and centrofacial and malar in 14\% of patients. Hyperpigmentation as confluent macules was seen in $86 \%(286 / 331)$ of the patients (north $92 \%$, east $91 \%$, south $88 \%$ and west $63 \%)$. The northern region showed a higher prevalence of centrofacial (55\%) and mandibular (5\%) patterns of melasma as compared to the other regions. The western (50\%) and eastern (48\%) regions demonstrated a higher prevalence of malar pattern melasma. An analysis of melasma pattern and regions showed a statistically significant $(p=0.0004)$ association. Multiple patterns of melasma were observed in the southern region as compared to any other region. In addition, the association between the pattern of melasma and gender was

Table 1 Demographic details

\begin{tabular}{llllllc}
\hline Location & East & West & North & South & Overall & $\boldsymbol{p}$ value \\
\hline Sample size $(n)$ & 44 & 82 & 78 & 127 & 331 & \\
Age (years, mean \pm SD) & $34.3 \pm 8.9$ & $38.6 \pm 7.7$ & $40.2 \pm 9.2$ & $35.4 \pm 9.9$ & $37.2 \pm 9.3$ & $0.0003^{*}$ \\
Sex (M/F) & $(10 / 34)$ & $(13 / 69)$ & $(13 / 65)$ & $(29 / 98)$ & $(65 / 266)$ & $0.5604^{*}$ \\
Marital status (married/single) & $(23 / 21)$ & $(69 / 13)$ & $(68 / 10)$ & $(97 / 30)$ & $(257 / 74)$ & $<0.001^{*}$ \\
Family history (Yes; \%) & $8(18.18)$ & $23(28.04)$ & $30(38.46)$ & $42(33)$ & $103(31.11)$ & $0.1207^{*}$ \\
\hline
\end{tabular}

$n$ number of patients, $S D$ standard deviation

* Chi-square test

* One-way ANOVA 


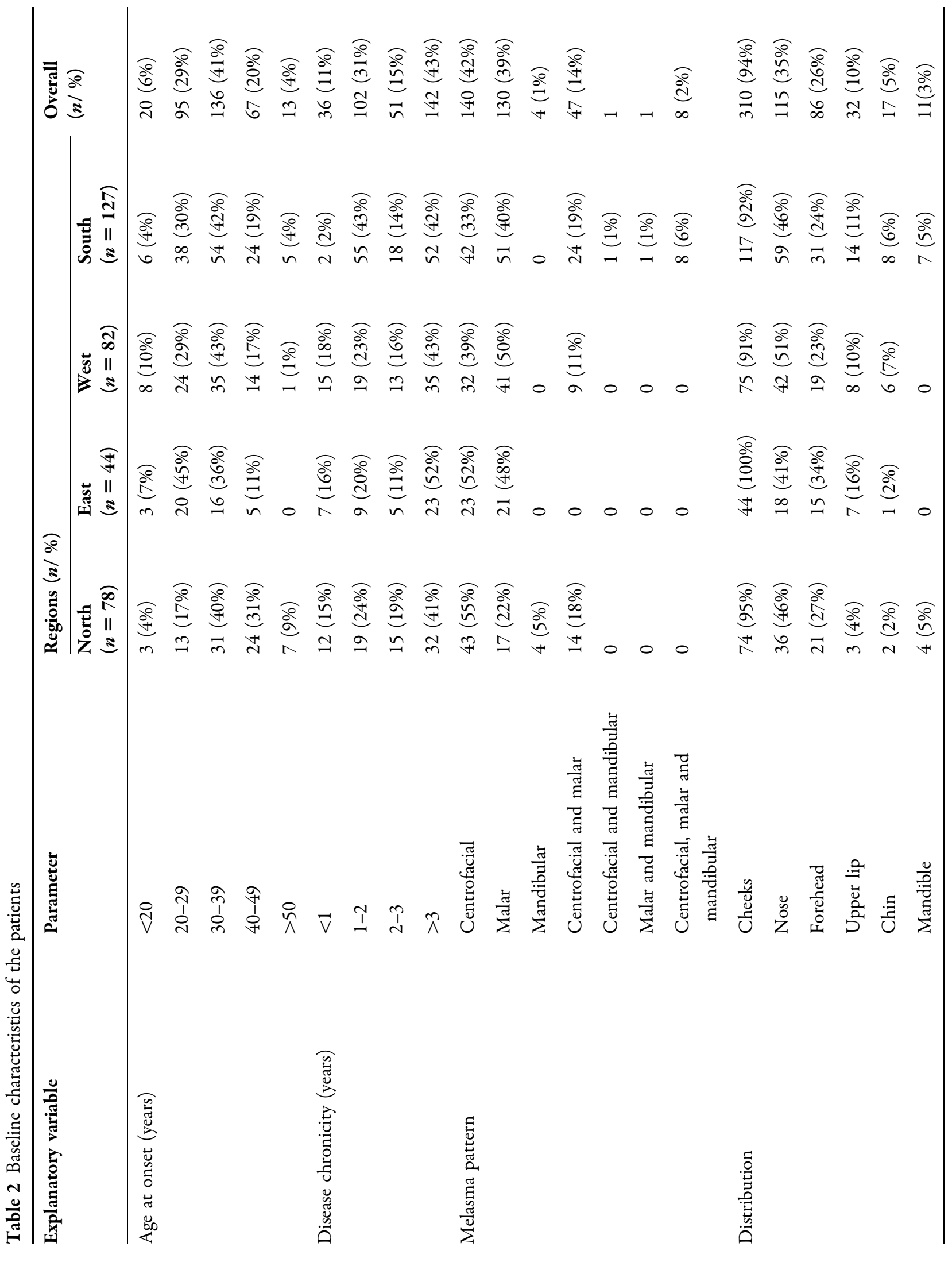




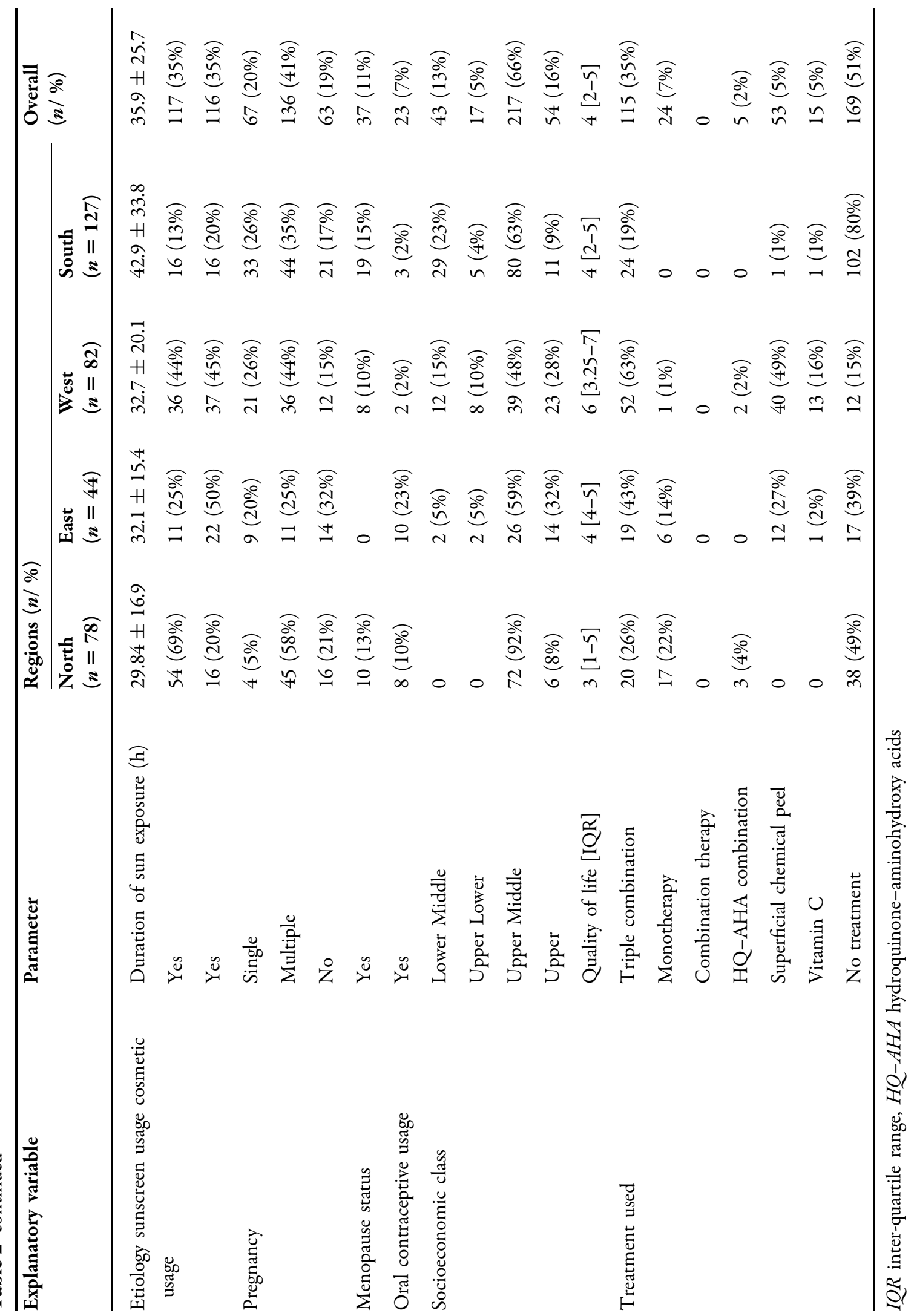


statistically significant ( $p=0.0369)$ with higher female patients (91\%) experiencing multiple patterns of melasma as compared to male patients.

\section{Effect of Other Variables on Prevalence of Melasma}

A total of 70\% patients were exposed to sun during the morning, and fewer patients were exposed to sun in the afternoon (29\%) and evening (4\%). The average duration of sun exposure per week across all regions was $35.9 \pm 25.7 \mathrm{~h}$. The average duration of sun exposure was higher in the southern region $(42.9 \pm 33.7 \mathrm{~h})$ when compared with other regions. The association of duration of sun exposure with gender was statistically significant $(p=0.0105)$ with males having higher sun exposure duration as compared to females (males $1.8 \pm 1.3$ days; females $1.4 \pm 0.9$ days).

The usage of sun protection was also recorded. Overall, only 35\% (117/331) of the patients were using sunscreens. The usage of sunscreens was observed to be highest in the northern region (69\%) followed by the western (44\%) region. Overall, $87 \%$ of the patients from the southern region reported no use of sunscreen. A statistically significant $(p<0.05)$ relationship was observed between sunscreen usage and regions. Among the patients using sunscreen, only $10 \%$ of the patients reported using a sun protection factor (SPF) $>50$. Among the patients using sunscreens, almost $73 \%$ of the patients reported having used sunscreens daily. The use of cosmetics was observed in 35\% of the overall population. Cosmetics were used either occasionally (1\%), two times a week (3\%) or three times a week (2\%). The highest use of cosmetics was observed in the eastern region (50\%), western region (45\%) and southern region $(32 \%)$.
The association between the pattern of melasma and cosmetic usage was evaluated. Multiple patterns of melasma (40\%) were observed in a higher proportion of patients as compared to a single pattern of melasma (34\%) among patients using cosmetics.

Of the 266 female patients who participated in the study, $23(8.6 \%)$ patients reported the use of oral contraceptives. Of the patients on oral contraceptives, 19 had a history of oral contraceptive use since 1-3 years and 7 of these patients also reported a history of melasma for 1-3 years. However, a statistically significant relationship could not be established due to a smaller sample size. But, the possibility of oral contraceptives precipitating melasma in $2.6 \%(7 / 266)$ women cannot be ruled out (Fig. 1).

The association of other conditions with the prevalence of melasma and disease chronicity revealed that a higher proportion of hypertension cases $(80 \%)$ and thyroid disease (74\%) were associated with a chronicity of more than 3 years. A statistically significant $(p<0.05)$ association was observed between the socioeconomic status of the patient and disease chronicity. A higher proportion of patients belonging to the lower middle class (56\%) had a chronicity of $>3$ years as compared to the other three groups (40-47\%).

About $51 \%$ of the women with multiple pregnancies had a history of melasma as compared to women with single $(25 \%)$ or no pregnancy (24\%). Thus, there is a possibility that pregnancy might be a precipitating factor in melasma. Of 37 patients who had achieved menopause, only 5 had a relapse.

Overall, $51 \%$ of the patients had not received any treatment for melasma. However, $71 \%$ of the patients who had tried previous treatments had used triple combination with the majority having used a mometasone-based triple 


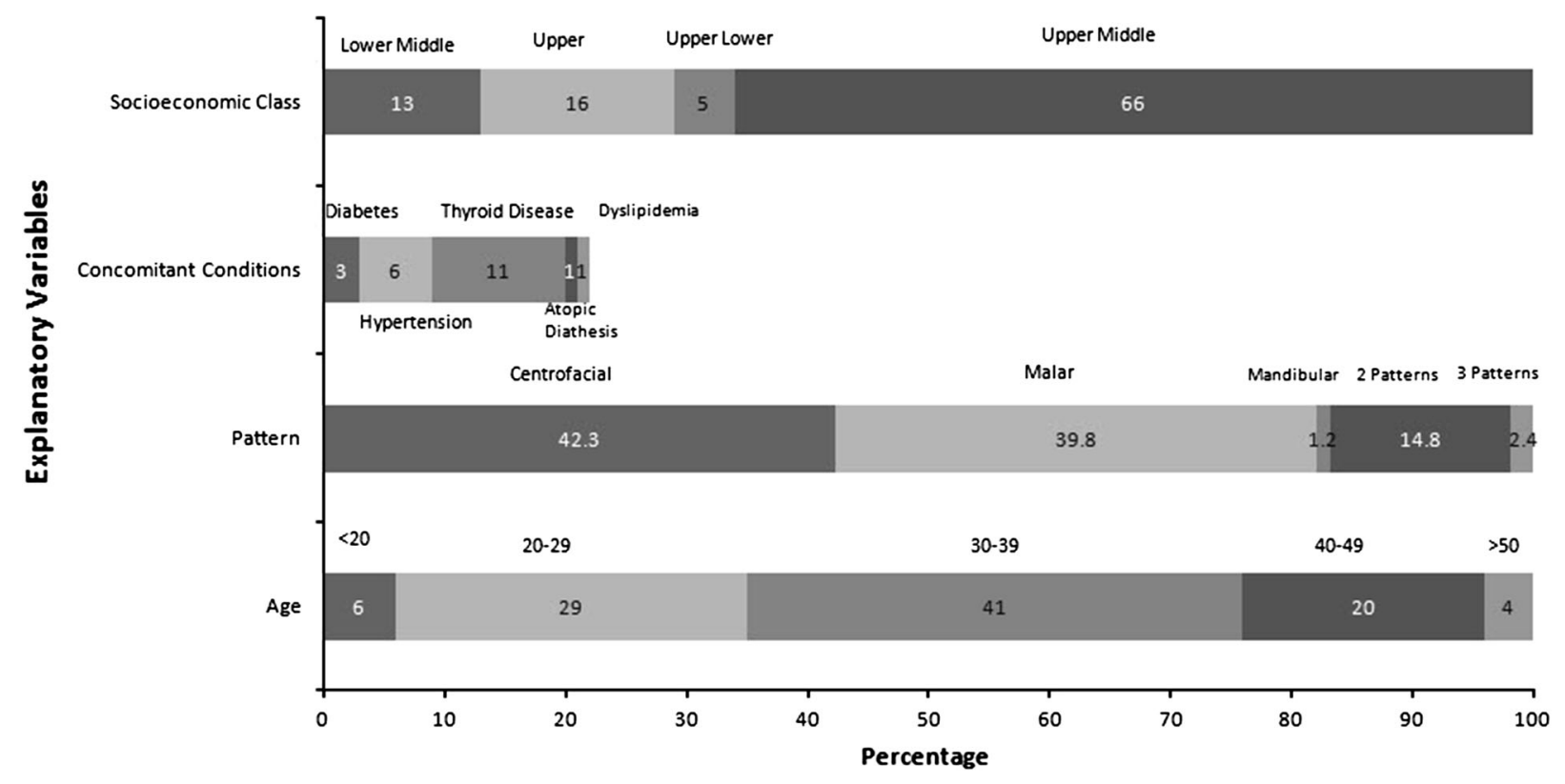

Fig. 1 Effect of various explanatory variables on prevalence of melasma

combination. The use of triple combinations was highest in the southern region followed by the western region. Clinical evaluation of these patients revealed telangiectasia (7\%) and exogenous ochronosis (3\%).

\section{DISCUSSION}

Melasma is a hyperpigmentation disorder that occurs on the sun-exposed areas of the skin. Hyperpigmentation appears on the face and neck; however, it may also occur in other locations. Caucasian women and individuals of darker complexion are more prone to this disorder $[12,13]$. Melasma has been reported in 8.8\% of Latin American females in the southern USA and in up to $40 \%$ population in south-east Asia $[14,15]$.

Melasma is more prevalent among women. There is no documented literature for the overall prevalence of melasma in India. The present study demonstrated melasma in $24.4 \%$ men consistent with the results of previous studies that showed melasma in $19.9 \%$ [6] and $25.8 \%$ [16] of male patients of Indian origin. The female to male ratio in the present study was 4:1 with lesser variation across all the four regions included in the present study, similar to the previous studies conducted in India [6]. Studies outside India have reported a higher proportion of females with melasma. Sivayathorn et al. [14] reported a female to male ratio of $6: 1$ in a Malaysian population and 24:1in an Indonesian population.

The average age of melasma patients was $37.2 \pm 9.3$ years with statistically significant variation across four regions. The mean age of patients in the eastern region was 34.3 years, similar to a hospital-based study from the same region (33.4 years) [6]. The mean age of onset of melasma observed in the present study was 34.1 years; however, a previously conducted hospital-based study reported the age of onset to be 29.9 years [6]. The disease chronicity of 3 years was similar to a previously conducted hospital-based study [6]. A $p$ value very close to 
the threshold significance level (0.05) indicates that the association between the two could not be rejected and it may be in this case because of sampling limitations.

The present study demonstrated a positive family history of melasma in $31.1 \%$ patients with minimal variations across the four regions. A previously conducted study has reported a similar (33.3\%) proportion of positive family histories among patients with melasma [6]. In addition, a positive family history was reported in $20-70 \%$ patients in other studies from the past $[17,18]$. The presence of positive family history demonstrated a correlation with the occurrence of melasma; however, it showed no association with the regions.

Centrofacial and malar regions were predominantly observed among the patients included in the present study. The patterns exhibited regional variation as reported in the previous studies from India $[6,8]$. Mandibular pattern was observed only among patients from the northern region. The present study showed a positive association between the pattern of melasma and gender of the patients. Multiple patterns were predominant in females and single pattern was predominant in males.

Multiple causative factors have been implicated in the etiology of melasma with UV radiation being the single most important factor. Pathak et al. [19] reported that exposure to sunlight exacerbated melasma in $100 \%$ of patients. In Orientals, $72.4 \%$ patients reported exacerbation due to sun exposure [14]; however, a previously conducted study of Indian origin showed exacerbation in 55.1\% patients with melasma [6]. The use of sunscreen is effective in the prevention of melasma and in the enhancement of the efficacy of other topical therapies for melasma [20].
A previously conducted Indian study has demonstrated a greater correlation between pregnancy, use of oral contraceptives and melasma [6]. The present study demonstrated that multiple pregnancies and use of oral contraceptive pills might be precipitating factors for melasma. However, further studies are needed to ascertain these facts $[13,19]$.

The present study demonstrated melasma in $11 \%$ patients with thyroid disorder as compared to $6.4 \%$ in a previous study [6]. A further analysis in the present study revealed that the presence of thyroid disorder could be a precipitating factor in only $0.7 \%$ female patients.

Most patients included in the present study visited a dermatologist and received treatment for melasma. However, a large proportion of patients were unsatisfied with the treatment being received. Most patients received mometasone triple-based combination for melasma, but $49 \%$ of the patients were unsatisfied with the treatment. A regional variability was observed in the use of triple combination with the highest use in the western and eastern regions.

\section{CONCLUSION}

The result of the study showed that there is a regional variability in the demographics, clinical manifestations and factors that precipitate melasma among patients in India. There was a strong correlation between the family history and prevalence of melasma. Sun exposure is a major precipitating factor in melasma, but only $10 \%$ of the patients used sunscreen with SPF $>50$. Other factors such as concomitant medication, chronicity of disease, multiple pregnancies and use of oral contraceptives might precipitate melasma. The 
sample size of the present study has limited the ability to find the association between some of the factors such as pregnancy, oral contraceptive pills usage and concomitant conditions with melasma. Further evaluation in a large patient pool will be useful.

\section{ACKNOWLEDGMENTS}

Sponsorship and article-processing charges for this study were funded by Wockhardt Ltd.

Medical writing assistance for this study was provided by Gurpreet Virya of Knowledge Isotopes Pvt. Ltd. (Mohali, Punjab, India) and was funded by Wockhardt Ltd (Mumbai, India). All named authors meet the ICMJE criteria for authorship for this manuscript, take responsibility for the integrity of the work as a whole and have given final approval for the version to be published.

Compliance with ethics. The study protocol and all the amendments were reviewed and approved by an independent institutional ethics committee/institutional review board (IEC/IRB). The study was conducted in accordance with good clinical practice (GCP) as required by the International Conference on Harmonization (ICH) guidelines and adopted in Schedule Y (Amended 2005) and also followed the Helsinki Declaration of 1975, as revised in 2000 and 2008. Informed consent was obtained from all patients for being included in the study.

Conflict of interest. D.S.R. KrupaShankar received research funding for the conduct of this trial from Wockhardt Ltd., Mumbai.

V.K. Somani received research funding for the conduct of this trial from Wockhardt Ltd., Mumbai.
M. Kohli received research funding for the conduct of this trial from Wockhardt Ltd., Mumbai.

J. Sharad received research funding for the conduct of this trial from Wockhardt Ltd., Mumbai.

A. Ganjoo received research funding for the conduct of this trial from Wockhardt Ltd., Mumbai.

S. Kandhari received research funding for the conduct of this trial from Wockhardt Ltd., Mumbai.

V.R. Mysore received research funding for the conduct of this trial from Wockhardt Ltd., Mumbai.

S. Aurangabadkar received research funding for the conduct of this trial from Wockhardt Ltd., Mumbai.

S. Malakar received research funding for the conduct of this trial from Wockhardt Ltd., Mumbai.

M. Vedamurthy received research funding for the conduct of this trial from Wockhardt Ltd., Mumbai.

S. Motlekar is an employee and stake holder by means of salary at Wockhardt Ltd., which sponsored the study.

P. Ahirrao is an employee and stake holder by means of salary at Wockhardt Ltd., which sponsored the study.

G. Kadhe is an employee and stake holder by means of salary at Wockhardt Ltd., which sponsored the study.

Open Access. This article is distributed under the terms of the Creative Commons Attribution Noncommercial License which permits any noncommercial use, distribution, and reproduction in any medium, provided the original author(s) and the source are credited. 


\section{REFERENCES}

1. Kang HY, Ortonne JP. What should be considered in treatment of melasma. Ann Dermatol. 2010;22:373-8.

2. Ting PT, Barankin B. Can you identify this condition? Can Fam Phys. 2005;51(353-355):353.

3. Estrada CR, Torres BB, Alarcon HH. Epidemiologia cutanea en dos sectores de atencion medica en Guerrero, Mexico. Dermatologia Rev Mex. 1992;36:29-34.

4. Failmezger C. Incidence of skin disease in Cuzco, Peru. Int J Dermatol. 1992;31(8):560-1.

5. Pasricha JS, Khaitan BK, Dash S. Pigmentary disorders in India. Dermatol Clin. 2007;25(3): 343-52 viii.

6. Achar A, Rathi SK. Melasma: a clinicoepidemiological study of 312 cases. Indian J Dermatol. 2011;56(4):380-2.

7. Sarvjot V, Sharma S, Mishra S, Singh A. Melasma: a clinicopathological study of 43 cases. Indian J Pathol Microbiol. 2009;52(3):357-9.

8. Thapa DM. Melasma (chloasma): a review with current treatment options. Indian J Dermatol. 2004;49:165-76.

9. Adalatkhah H, Sadeghi-bazargani H, Amini-sani N, Zeynizadeh S. Melasma and its association with different types of nevi in women: a case-control study. BMC Dermatol. 2008;8:3.

10. Misery L, Schmitt AM, Boussetta S, Rahhali N, Taieb C. Melasma: measure of the impact on quality of life using the French version of MELASQOL after cross-cultural adaptation. Acta Derm Venereol. 2010;90(3):331-2.
11. Pichardo R, Vallejos Q, Feldman SR, Schulz MR, Verma A, Quandt SA, et al. The prevalence of melasma and its association with quality of life in adult male Latino migrant workers. Int J Dermatol. 2009;48(1):22-6.

12. Grimes PE. Melasma. Etiologic and therapeutic considerations. Arch Dermatol. 1995;131(12): 1453-7.

13. Sanchez NP, Pathak MA, Sato S, Fitzpatrick TB, Sanchez JL, Mihm MC Jr. Melasma: a clinical, light microscopic, ultrastructural, and immunofluorescence study. J Am Acad Dermatol. 1981;4(6):698-710.

14. Sivayathorn A. Melasma in orientals. Clin Drug Invest. 1995;10:34-40.

15. Werlinger KD, Guevara IL, Gonzalez CM, Rincon ET, Caetano R, Haley RW, et al. Prevalence of selfdiagnosed melasma among premenopausal Latino women in Dallas and Fort Worth, Tex. Arch Dermatol. 2007;143(3):424-5.

16. Sarkar R, Jain RK, Puri P. Melasma in Indian males. Dermatol Surg. 2003;29(2):204.

17. Resnik S. Melasma induced by oral contraceptive drugs. JAMA. 1967;199(9):601-5.

18. Vazquez M, Maldonado H, Benmaman C, Sanchez JL. Melasma in men. A clinical and histologic study. Int J Dermatol. 1988;27(1):25-7.

19. Pathak MA. Clinical and therapeutic aspects of Melasma: an overview. In: Fitz Patrick TB, Wick MM, Toda K, editors. Brown melanoderma Tokyo: University of Tokyo Press; 1986. p. 161-72.

20. Sheth VM, Pandya AG. Melasma: a comprehensive update: part II. J Am Acad Dermatol. 2011;65(4):699-714 quiz 5. 